\title{
Estimation des coefficients de frottement et de dissipation d'énergie dans la houle au voisinage d'un fond de rides
}

\author{
F. Marin* \& J. Brossard** \\ Maîtres de Conférences, Laboratoire de Mécanique, Université du Havre, \\ * B.P. 4006, 76610 Le Havre, \\ *: B.P. 540, 76058 Le Havre Cedex.
}

Résumé

Nous avons réalisé des mesures de vitesse par Vélocimétrie Doppler Laser deux composantes dans un canal à houle au-dessus d'un fond ridé fixe afin d'estimer les coefficients de frottement $f_{w}$ et de dissipation d'énergie $f_{c}$ au fond. Les coefficients $\mathrm{f}_{\mathrm{w}}$ ont été déterminés par l'approche classique de l'intégrale de quantité de mouvement en prenant en compte l'effet du gradient de pression sur la surface des rides et à partir des tensions de Reynolds. Les résultats obtenus par les deux méthodes sont voisins et suggèrent que les modèles théoriques de Jonsson [3] et de Kamphuis [6] permettent une bonne estimation de $f_{w}$. Les coefficients $f_{c}$ apparaissent sous-estimés par les modèles de Blondeaux et Vittori [1] et de Sleath [9].

\section{Introduction}

Lorsqu'une houle se propage en direction d'une côte, la plupart de la dissipation de son énergie en dehors de la zone de déferlement s'effectue dans la couche limite de fond. Cette dissipation d'énergie est directement liée aux forces horizontales agissant sur les sédiments composant le fond. La détermination des coefficients de frottement et de dissipation d'énergie dans la houle sont d'une grande utilité en transport sédimentaire. Ces coefficients peuvent notamment être estimés à partir de l'approche classique de l'intégrale de quantité de mouvement où à partir des tensions de Reynolds mesurées. Les études précédemment réalisées sur ce sujet (Jonsson [3], Kamphuis [6], Sleath [9,12],...) montrent les différences importantes entre les estimations de ces coefficients suivant l'approche utilisée. Ainsi, les valeurs des coefficients de frottement obtenues par Sleath [12] different d'un facteur 10 suivant la méthode utilisée.

Le but de cette étude est d'obtenir une estimation plus précise des coefficients de frottement et de dissipation d'énergie de la houle au voisinage d'un fond de rides à partir de mesures de vitesse effectuées dans un canal à houle par Vélocimétrie Doppler Laser (VDL) 2 composantes. Pour cela, les mesures ont été réalisées sur une longueur d'onde de ride avec un maillage très fin du fond jusqu'à l'extérieur de la couche limite. L'effet très souvent négligé du gradient de pression dans le fluide agissant sur la surface des rides a été pris en compte dans l'estimation de $f_{w}$, ce gradient dépendant de la géométrie des rides, de la période de l'écoulement ainsi que de l'amplitude de la vitesse des particules fluides juste à l'extérieur de la couche limite de fond. 


\section{Dispositif expérimental}

Les études ont été réalisées à l'Université du Havre dans un canal à houle de $9 \mathrm{~m}$ de long, 0,8 $\mathrm{m}$ de large et $1 \mathrm{~m}$ de hauteur. Ce canal est muni de parois latérales en verre afin de pouvoir réaliser aisément des mesures par VDL. La houle est générée par un batteur plan disposé à une extrémité du canal. Un plateau à excentrique permet le réglage de l'excursion de la pale et donc de l'amplitude de la houle. Une plage d'amortissement située à l'autre extrémité du canal permet d'obtenir un coefficient de réflexion de l'ordre de $5 \%$ pour les conditions de houle utilisées.

La hauteur d'eau moyenne d a été fixée à $27 \mathrm{~cm}$ et la période de la houle $T$ à $1,08 \mathrm{~s}$. Un test préliminaire a été réalisé avec un fond plan lisse afin de vérifier que la valeur du coefficient de frottement obtenue expérimentalement correspond bien à la valeur théorique. Nous avons ensuite utilisé un fond ridé artificiel dont la géométrie peut être décrite par le système d'équations paramétriques classique (Sleath [10]) suivant:

$$
\left\{\begin{array}{l}
x=X-\frac{h}{2} \sin \left(\frac{2 \pi X}{L}\right) \\
y=\frac{h}{2} \cos \left(\frac{2 \pi X}{L}\right)
\end{array}\right.
$$

avec: $h=3 \mathrm{~mm}$, hauteur (crête-creux) des rides,

$\mathrm{L}=18 \mathrm{~mm}$, longueur d'onde des rides $\left(\frac{\mathrm{h}}{\mathrm{L}}=0,17\right)$,

$(\mathrm{x}, \mathrm{y})$ : système orthogonal de coordonnées dont l'axe des $\mathrm{x}$ est parallèle au fond et dirigé dans le sens de propagation de la houle et l'axe des y orienté vers le haut; l'origine de ce repère est située sur la verticale du sommet de la ride considérée à mi-hauteur de ride, $\mathrm{X}$ : paramètre de l'équation.

Les rides sont ainsi symétriques à crêtes arrondies, le rayon de courbure étant plus faible au sommet qu'au creux.

Plus de 1200 mesures de vitesse par VDL 2 composantes ont été effectuées audessus d'une ride, de la surface de la ride jusqu'à l'extérieur de la couche limite, soit approximativement sur $15 \mathrm{~mm}$ de hauteur. Le pas des mesures était de 0,25 $\mathrm{mm}$ dans le creux de la ride et de $0,5 \mathrm{~mm}$ au-dessus, le volume de mesure étant de $0,14 \mathrm{~mm}^{3}$. Les conditions expérimentales sont précisées dans le tableau 1. Dans ce tableau, $\mathrm{H}$ est la hauteur des vagues, $\mathrm{u}_{\infty}$ l'amplitude de la composante horizontale de la vitesse juste à l'extérieur de la couche limite de fond, a l'amplitude des trajectoires des particules fluides au même endroit et $R$ le nombre de Reynolds basé sur $u_{\infty}$ et a $\left(R=\frac{u_{\infty} a}{v}\right.$ avec $v$ : viscosité cinématique). Ces conditions induisent une houle pouvant être modélisée par la théorie de Stokes développée au $2^{\text {ime }}$ ordre pour le test $\mathrm{N}^{\circ} 1$ et au $3^{\text {ime }}$ ordre pour le test $\mathrm{N}^{\circ} 2$. 


\begin{tabular}{ccccccc}
\hline Test $\mathrm{N}^{\circ}$ & $\mathrm{d}(\mathrm{mm})$ & $\mathrm{T}(\mathrm{s})$ & $\mathrm{H}(\mathrm{mm})$ & $\mathrm{u}_{\infty}(\mathrm{m} / \mathrm{s})$ & $\mathrm{a}(\mathrm{m})$ & $\mathrm{R}$ \\
\hline 1 & 270 & 1,08 & 48 & 0,099 & 0,0171 & 1700 \\
2 & 270 & 1,08 & 92 & 0,176 & 0,0302 & 5300 \\
\hline
\end{tabular}

Tableau 1 : Conditions expérimentales.

La taille des rides a été choisie de façon à reproduire les rides qu'on aurait observé avec un fond sableux pour les conditions de houle du test $\mathrm{N}^{\circ} 1$. Pour ce test, le régime de l'écoulement est transitoire alors qu'il est turbulent pour le test $\mathrm{N}^{\circ} 2$ selon Kajiura [5].

\section{Contraintes de cisaillement en écoulement oscillant}

En négligeant les termes non linéaires autres que les tensions de Reynolds, l'équation de quantité de mouvement pour un écoulement au-dessus d'un fond horizontal peut s'écrire:

$$
\frac{\partial u}{\partial t}=-\frac{1}{\rho} \frac{\partial p}{\partial x}+\frac{1}{\rho} \frac{\partial \tau}{\partial y}
$$

où $u, t, \rho, p$ et $\tau$ représentent respectivement la composante horizontale de la vitesse, le temps, la masse volumique, la pression et la contrainte de cisaillement. Cette contrainte étant nulle à l'extérieur de la couche limite de fond, nous avons:

$$
\frac{\partial u_{0}}{\partial t}=-\frac{1}{\rho} \frac{\partial p}{\partial x}
$$

avec $u_{0}$ : valeur de u juste à l'extérieur de la couche limite de fond. L'équation (2) peut donc se mettre sous la forme:

$$
\tau=\rho \int_{y}^{\infty} \frac{\partial\left(u_{0}-u\right)}{\partial t} d y
$$

Dans le cas d'un fond ridé dont le profil est décrit par les équations (1), considérons le système de coordonnées orthogonales $(\xi, \eta)$ défini par (Sleath, [8]):

$$
\left\{\begin{array}{l}
\xi=x+\frac{h}{2} \exp (-k \eta) \sin (k \xi) \\
\eta=y-\frac{h}{2} \exp (-k \eta) \cos (k \xi)
\end{array}\right.
$$

avec $k=\frac{2 \pi}{L}$. Le fond est alors représenté par la ligne $\eta=0$. 
Soit un volume de contrôle contenant une longueur d'onde de ride et s'étendant du fond à l'infini. Supposons que ce volume s'étende d'une crête de ride à une autre. La force horizontale agissant sur le fluide dans ce volume résulte de la force horizontale exercée par le fond sur le fluide et des forces de pression exercées aux deux extrémités (amont et aval). Les vitesses des particules fluides peuvent être supposées identiques (en moyenne) aux deux extrémités et la résultante de ces forces de pression peut s'écrire: $\int_{\frac{h}{2}}^{\infty} L \frac{\partial p}{\partial x} d y$. Ainsi, la force par unité de largeur $F$ exercée par le fluide sur une longueur d'onde du fond est:

$$
F=-\rho \frac{\partial}{\partial t} \int_{x=0}^{L} \int_{\eta=0}^{\infty} u d y d x-L \frac{\partial p}{\partial x} \int_{\frac{h}{2}}^{\infty} d y
$$

En supposant que la vitesse juste à l'extérieur de la couche limite obéisse à la loi:

$$
\mathrm{u}_{0}=\mathrm{u}_{\infty} \cos (\omega \mathrm{t})
$$

où $\omega=\frac{2 \pi}{\mathrm{T}}$, nous obtenons:

$$
F=\rho \int_{x=0}^{L} \int_{\eta=0}^{\infty} \frac{\partial}{\partial t}\left(u_{0}-u\right) d y d x+\frac{h}{2} L \rho u_{\infty} \omega \sin (\omega t)
$$

Pour déterminer $F$, il suffit d'effectuer le calcul sur un volume de contrôle allant du fond à l'extérieur de la couche limite de fond. Les coefficients de frottement à la paroi $f_{w}$ et de dissipation d'énergie $f_{c}$ peuvent être estimés en appliquant les formules de Jonsson [4]:

$$
\begin{aligned}
& f_{w}=\frac{(F / L)_{\max }}{0,5 p u_{\infty}^{2}} \\
& f_{e}=\frac{3 \pi \overline{u_{0} F / L}}{2 p u_{\infty}^{3}}
\end{aligned}
$$

la moyenne temporelle (barre horizontale) dans (10) étant faite sur une période de la houle.

Nous utiliserons également une autre approche qui consiste à négliger les effets de la viscosité moléculaire et à estimer la contrainte de cisaillement à partir des tensions de Reynolds:

$$
\tau=\frac{F}{L}=-\overline{u^{\top} v^{2}}
$$


où v représente la composante verticale de la vitesse et les primes les composantes turbulentes de la vitesse.

\section{Analyse des résultats}

Nous avons tout d'abord effectué un profil de vitesse au-dessus d'un fond lisse pour lequel les valeurs du coefficient de frottement sont bien connues. La période et l'amplitude de la houle étaient respectivement de $1,08 \mathrm{~s}$ et $14 \mathrm{~mm}$ et l'écoulement laminaire. Le coefficient de frottement $\mathrm{f}_{w}$ obtenu avec les relations (4) et (9) est de 0,055 alors que la valeur théorique donnée par:

$$
\mathrm{f}_{\mathrm{w}}=\frac{2}{\sqrt{\mathrm{R}}}
$$

est de 0,060 (R=1100). L'accord entre la valeur théorique et la valeur expérimentale est satisfaisant.

Dans le cas du fond ridé, la figure 1 donne l'évolution avec le temps de la force $\mathrm{F}$ par unité de largeur exercée par le fluide sur une longueur d'onde du fond, F étant ici déterminée à partir de la relation (8). Pour le test $\mathrm{N}^{\circ} 2(\mathrm{R}=5300)$, la rupture de pente à $\omega t \approx 4$ peut provenir de l'intégration suivant $\mathrm{x}$ effectuée pour le calcul de F (équation (8)).

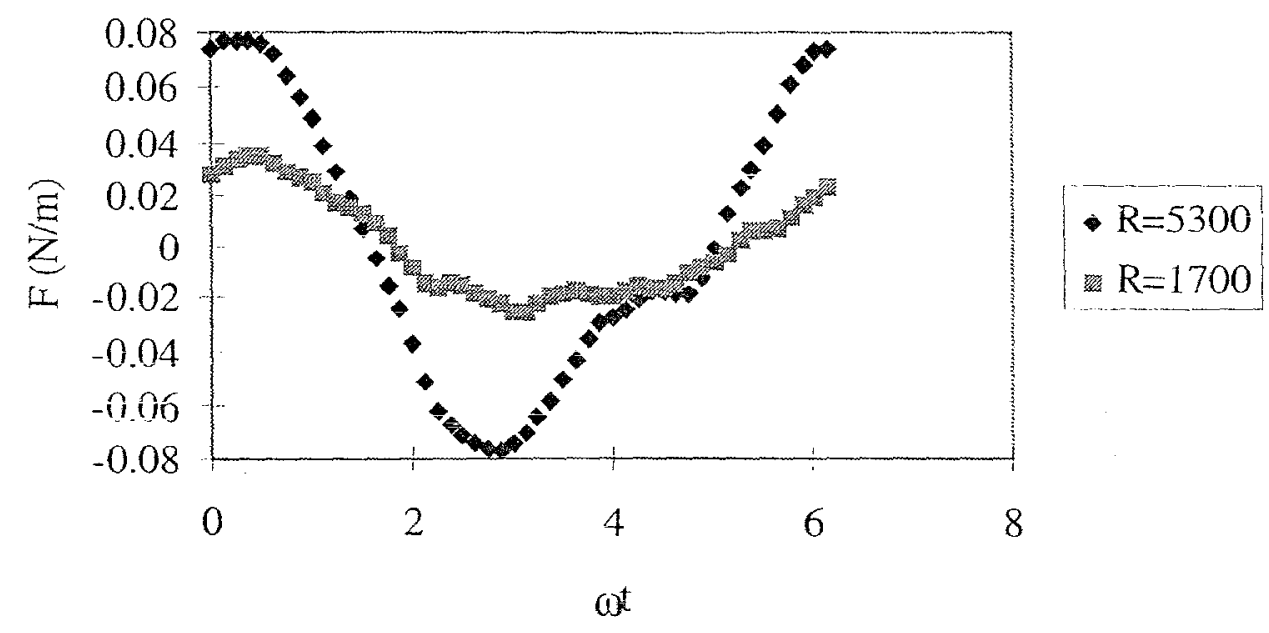

Figure 1. Evolution temporelle de $\mathrm{F}$ pour $\mathrm{R}=1700$ et $\mathrm{R}=5300$.

Les valeurs obtenues pour $f_{w}$ avec la relation (9) sont représentées sur la figure 2 ("Tests réalisés, méthode intégrale"). La rugosité équivalente $\mathrm{k}_{\mathrm{s}}$ des rịdes a été calculée avec la formule de Swart [13]:

$$
\frac{k_{s}}{h}=25\left(\frac{h}{L}\right)
$$

Déterminons maintenant la contrainte de cisaillement à partir des tensions de Reynolds. La variation avec y des valeurs maximales moyennées sur une longueur d'onde de ride des tensions de Reynolds est donnée sur la figure 3. 


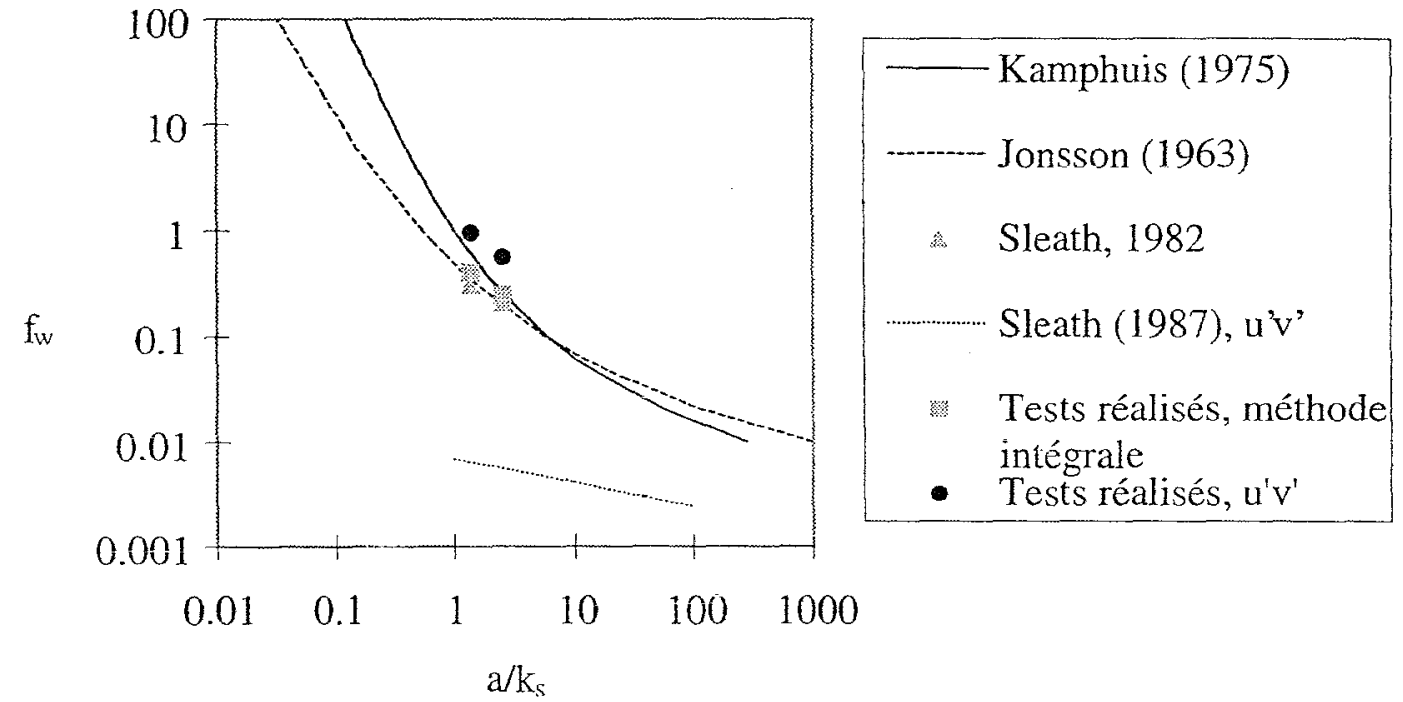

Figure 2. Valeurs des coefficients de frottement.

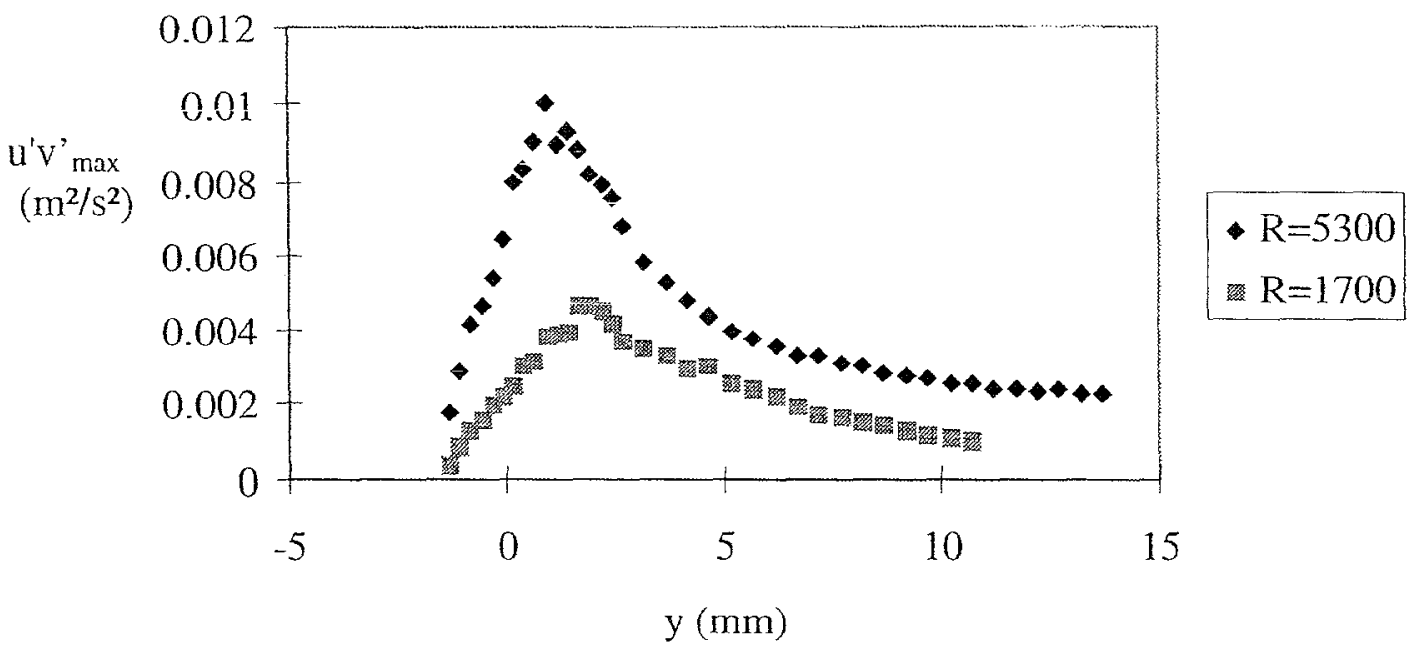

Figure 3. Variation des valeurs maximales moyennées sur une longueur d'onde de ride des tensions de Reynolds.

L'amplitude des tensions de Reynolds évolue de façon classique: elle augmente jusqu'à un maximum puis décroît régulièrement avec la distance par rapport au fond. En suivant l'hypothèse traditionnelle suivant laquelle la valeur maximale de la tension de Reynolds est égale à la contrainte de cisaillement au fond, nous obtenons les valeurs de $f_{w}$ représentées sur la figure 2 ("Tests réalisés, $u^{\prime} v^{\prime}$ "). Nous avons également représenté sur cette figure la courbe empirique de Kamphuis [6], la courbe semi-empirique de Jonsson [3] et les résultats du modèle de Sleath [9] pour les valeurs de $\frac{\mathrm{a}}{\mathrm{k}_{\mathrm{s}}}$ correspondant à celles de notre étude. Ce modèle utilise une solution aux différences finies de l'équation bidimensionnelle de vorticité. Sleath [12] a montré que dans le cas d'un fond plat rugueux, il obtenait des valeurs 
de $f_{w}$ très différentes suivant qu'il utilisait la relation (4) ou la relation (11). En effet, ses résultats sont en bon accord avec les courbes de Kamphuis et de Jonsson Iorsqu'il utilise la relation (4) alors qu'il obtient des valeurs approximativement 10 fois plus faibles à partir des tensions de Reynolds (relation 11) comme on peut le voir sur la figure 2 ("Sleath (1987), u'v"'). Ces dernières valeurs apparaissent sous estimées. Les résultats que nous obtenons en prenant en compte l'effet du gradient de pression sur le fond ridé ne diffèrent que d'un facteur 2 avec la méthode appliquée et sont très proches des courbes de Kamphuis [6] et Jonsson [3] ainsi que des valeurs résultant du modèle de Sleath [9]. Les formules proposées par ces trois auteurs semblent ainsi bien adaptées pour estimer le coefficient de frottement $f_{w}$ au niveau d'un fond de ride subissant l'action de la houle dans la plage de variation de $\frac{\mathrm{a}}{\mathrm{k}_{\mathrm{s}}}$ étudiée, c'est-à-dire pour $1 \leq \frac{\mathrm{a}}{\mathrm{k}_{\mathrm{s}}} \leq 3$. Ceci n'est en fait pas une limitation significative puisque les fonds ridés naturels sont généralement caractérisés par des valeurs $\frac{a}{k_{\mathrm{s}}}$ de l'ordre de 1 , excepté pour des rides de pente très faible.

Les coefficients de dissipation d'énergie $\mathrm{f}_{\mathrm{c}}$ définis par la relation (10) sont donnés figure 4.

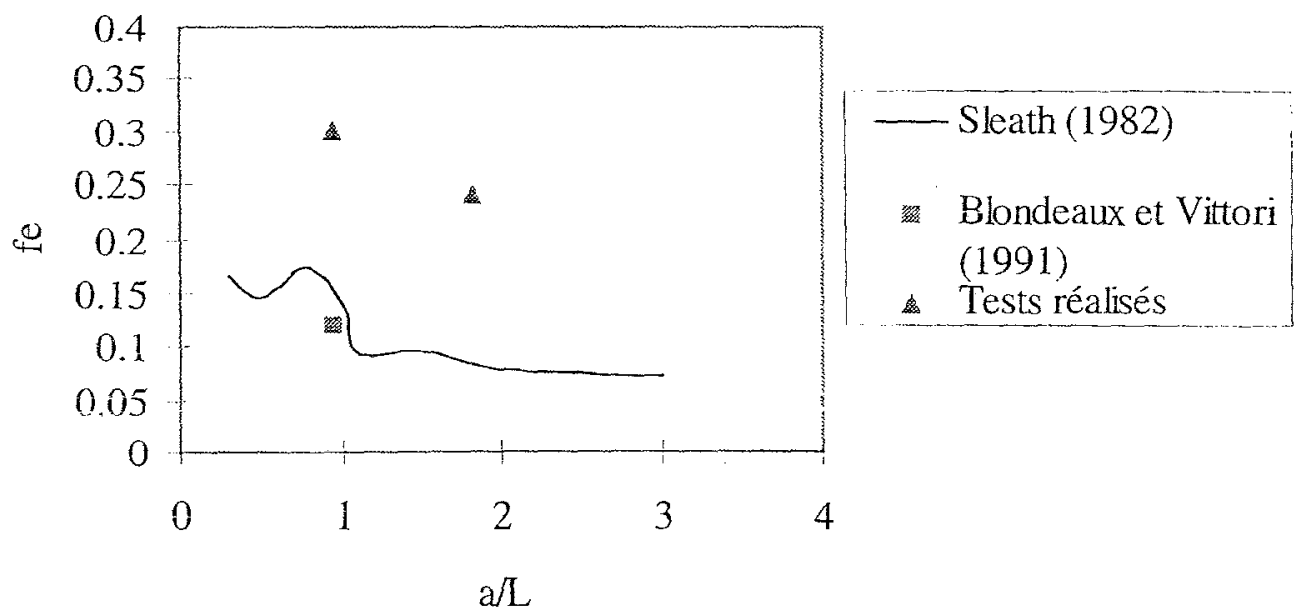

Figure 4. Evolution de $\mathrm{f}_{\mathrm{c}}$ avec a/L.

Les valeurs que nous obtenons sont approximativement deux fois supérieures aux résultats des modèles de Sleath [9] et Blondeaux et Vittori [1]. Ce dernier modèle résout les équations de vorticité et de Poisson pour la fonction de courant à partir de méthodes spectrales et d'approximations aux différences finies. La sousestimation de $\mathrm{f}_{\mathrm{e}}$ par les modèles peut être en partie due au fait que ces módèles ne prennent pas en compte la turbulence dans l'écoulement. Carstens et al. [2] et Lofquist [7] ont effectué des mesures de dissipation d'énergie au-dessus d'un fond ridé mobile sableux. La grande dispersion des résultats et le fait que la rugosité du sable ainsi que le mouvement du fond contribuent à la valeur de $f_{c}$ ne permettent pas une comparaison significative des résultats. 


\section{Conclusion}

Cette étude réalisée en canal et basée sur des mesures de vitesse par VDL 2 composantes nous a permis de déterminer expérimentalement les coefficients de frottement et de dissipation d'énergie au niveau d'un fond ridé fixe subissant l'action de la houle. L'effet du gradient de pression dans le fluide agissant sur la surface des rides a été pris en compte et les résultats obtenus à partir de l'approche classique de l'intégrale de quantité de mouvement d'une part et à partir des tensions de Reynolds d'autre part sont voisins. Les valeurs des coefficients de frottement $f_{w}$ sont en bon accord avec les modèles théoriques de Jonsson [3] et Kamphuis [6] alors que les modèles de Sleath [9] et de Blondeaux et Vittori [1] semblent sous estimer les coefficients de dissipation d'énergie $f_{e}$; ceci peut être dû en partie au fait que ces derniers modèles ne prennent pas en compte la turbulence dans l'écoulement.

\section{Références}

[1] Blondeaux P. et Vittori G. 1991. Vorticity dynamics in an oscillatory flow over a rippled bed. J. Fluid Mech., Vol. 226, pp. 257-289.

[2] Carstens M.R., Neilson F.M. et Altinbilek H.D. 1969. Beds forms generated in the laboratory under an oscillatory flow. U.S. Army Coastal Eng. Research Center, Tech. Memo. $\mathrm{N}^{\circ} 28$.

[3] Jonsson I.G. 1963. Measurements in the turbulent wave boundary layer. Proc. $10^{\text {th }}$ Congress IAHR, London, pp. 85-92.

[4] Jonsson I.G. 1980. A new approach to oscillatory rough turbulent boundary layers. Ocean Eng. 7, pp. 109-152.

[5] Kajiura K. 1968. A model of the bottom boundary layer in water waves. Bull. Earthquake Res. Inst. 46, pp. 75-123.

[6] Kamphuis J.W. 1975. Friction factors under oscillatory waves. Proc. A.S.C.E. J. Waterw. Harbors Coastal Eng. Div. 101 (WW2), pp. 135-144.

[7] Lofquist K.E.B. 1980. Measurements of oscillatory drag on sand ripples. Proc. $17^{\text {th }}$ Conf. Coastal Eng., Sydney, pp. 1-20.

[8] Sleath J.F.A. 1973. A numerical study of the influence of bottom roughness on mass transport by water waves. Proc. International Conf. Numerical methods in Fluid Dynamics, Southampton, pp. 1-10.

[9] Sleath J.F.A. 1982. Friction coefficients of rippled beds in oscillatory flow. Continental Shelf Research, Vol. 1, No1, pp. 33-47.

[10] Sleath J.F.A. 1984. Sea bed mechanics. Wiley-Interscience.

[11] Sleath J.F.A. 1985. Energy dissipation in oscillatory flow over rippled beds. Coastal Eng. 9, pp. 159-170.

[12] Sleath J.F.A. 1987. Turbulent oscillatory flow over rough beds. J. Fluid Mech., Vol. 182, pp. 369-409.

[13] Swart D.H. 1976. Coastal sediment transport. Computation of longshore transport. Delft Hydraul. Lab. Rep. R968. Part 1. 